Erschienen in: Stickel, Gerhard (Hrsg.): Varietäten des Deutschen.

Regional- und Umgangssprachen. - Berlin, New York: de Gruyter, 1997. S. 399-404. (Institut für deutsche Sprache. Jahrbuch 1996)

\title{
JAN GOOSSENS
}

\section{"Dialektverfall" und „Mundartrenaissance" in Westniederdeutschland und im Osten der Niederlande}

Das Dialektgebiet, von dem in diesem kleinen Beitrag die Rede ist, umfaBt das westliche niederdeutsche Gebiet und den angrenzenden Osten des niederländischen Sprachraums. Ich konzentriere mich dabei besonders auf die Situation in einem Streifen beiderseits der deutschniederländischen Staatsgrenze.

Das sprachhistorische Verhältnis der Dialekte diesseits und jenseits dieser Grenze ist bekannt. Sie gingen ineinander über; die wichtigsten Isoglossen im Grenzgebiet fielen nicht mit der Staatsgrenze zusammen und liefen nicht mit ihr parallel, sondern durchschnitten sie in der Quere. Seit dem vorigen und in diesem Jahrhundert übernehmen die Dialekte auf deutscher Seite immer mehr Elemente aus der deutschen, diejenigen auf der niederländischen Seite entsprechend aus der niederländischen Standardsprache. Diese Entwicklung ist am deutlichsten im Wortschatz zu beobachten, vor allem bei Bezeichnungen von Begriffen aus der technisierten und verwalteten Welt. Aber auch die Grammatik (Phonologie, Morphologie und Syntax) unterliegt diesem EinfluB. Das bedeutet nicht nur, daB die Mundarten auf deutscher Seite immer stärker "eingedeutscht" und die auf niederländischer "niederlandisiert" werden, sondern es bedeutet auch Dialektverfall, in dem Sinne nämlich, daß beide Gruppen Merkmale verlieren, die sie gegen die jeweilige Standardsprache absetzen, und Merkmale dazugewinnen, die sie mit ihr gemeinsam haben. AuBerdem werden sie so untereinander ähnlicher, was bedeutet, daß es zwischen ihnen weniger Unterschiede gibt, die als dialektal einzustufen sind. Diese Art des Dialektverfalls kann man als "Verwässerung” charakterisieren.

Dialektverfall gibt es auch in einem zweiten Sinn: Es wird immer weniger Dialekt gesprochen, von immer weniger Menschen in immer weniger Situationen. Anders formuliert: Formen der hochdeutschen bzw. der niederländischen Umgangssprache werden von immer mehr Menschen in 
immer mehr Situationen gesprochen. Diese Art des Dialektverfalls kann also als "Dialektverlust” charakterisiert werden. Der Dialektverlust ist mit den bekannten soziolinguistischen Parametern beschreibbar: Alter, Sozialschicht, Situation, AusmaB des Formalismus. Als sehr bedeutend erweist sich auch der geographische Faktor, wonach Gegensätze zwischen Stadt und Land, Zentrum und Peripherie, Gebieten mit viel und solchen mit wenig Immigration zu berücksichtigen sind. Die Beschreibung kann verfeinert werden mit Hilfe weiterer Faktoren wie Geschlecht und Domäne. All diese Faktoren korrelieren übrigens auch mit dem Grad der Dialektverwässerung. Innerhalb des gesamten Niederdeutschen haben die westlichen Grenzdialekte sich in der Vergangenheit als konservativ erwiesen, d.h. ihr Rückgang vollzog sich durchschnittlich weniger schnell als jener der mehr zentralen westfälischen und niedersächsischen Dialekte. In den letzten Jahrzehnten hat er sich aber stark beschleunigt: Heute gibt es kaum noch Familien, in denen die Kinder mundartlich sozialisiert werden. Auf niederländischer Seite läBt sich eine parallele Entwicklung beobachten. Die Problematik ist hier später thematisiert worden als auf deutscher Seite, doch können die folgenden beiden Beobachtungen als sicher gelten: Auf deutscher Seite ist die Entwicklung durchschnittlich weiter fortgeschritten (um 1 bis 3 Jahrzehnte), und die Streuung der umgangssprachlichen Abweichungen von einer standardsprachlichen Norm in den einzelnen Idiolekten ist geringer als auf niederländischer Seite. Das heißt, daß die Sprechweisen auf deutscher Seite deutlicher um zwei Pole kreisen, die sich auch deutlicher gegeneinander abheben als auf niederländischer Seite: Man spricht entweder die eine Sprache (Dialekt) oder die andere (Hochdeutsch). Sowohl im SprachbewuBtsein der Sprecher als bei der Analyse der sprachlichen Struktur der ÄuBerungen ist immer klar, bei welchem Pol man sich befindet. Auf niederländischer Seite sind die Übergänge fließender, wie offenbar im Süden des deutschen Sprachraums auch. Das dürfte mit einem geringeren strukturellen Abstand zwischen Dialekt und Standardsprache zusammenhängen, während in Norddeutschland die Mundartsprecher das Hochdeutsche sich zunächst als eine Art Fremdsprache aneignen mußten. Das erklärt auch, warum Erforscher der regionalen Varietäten im Osten der Niederlande im Gegensatz zu ihren niederdeutschen Kollegen das Bedürfnis haben, bestimmte Sprachformen, nämlich diejenigen, die ihre primären Dialektmerkmale und manchmal auch noch mehr verloren haben, mit einem eigenen Ausdruck zu bezeichnen: Man spricht von Regiolekten und von einer Regiolektisierung der Mundarten. Auch besteht hier ein größeres Interesse als auf deutscher Seite für die regionale Ausprägung der gesprochenen Formen der allgemeinen Sprache. 
Die Antwort auf die Alternativfrage, die den Teilnehmern an der Podiumsdiskussion der IDS-Tagung 1996 vorgelegt wurde, lautet somit für mein Gebiet eindeutig: Dialektverfall. Sie gründet sich auf zahlreiche Untersuchungen, die, was den Aspekt „Dialektverlust” betrifft, auf deutscher Seite seit der zweiten Hälfte der dreißiger Jahre, auf niederländischer seit den sechziger Jahren durchgeführt worden sind. Was den Aspekt „Dialektverwässerung” betrifft, hat die niederländische Forschung einen kleinen Vorsprung. Hier wird er seit den sechziger Jahren, auf deutscher Seite seit der zweiten Hälfte der siebziger Jahre thematisiert. Mehrere Studien, zuerst Kremer (1979), sind von vornherein vergleichend angelegt worden. Auffallig ist der große Anteil der Veröffentlichungen aus der Hand nichtprofessioneller Beobachter und auch der Veröffentlichungen in mehr oder weniger populären Medien. Das gilt vor allem für den Aspekt „Dialektverlust”.

Inwiefern muß nun meine Antwort relativiert werden? Erstens einmal gibt es die Erscheinung des sekundären Dialekterwerbs, vor allem in den sog. peer groups, von der mancher Mundartfreund sich ein zähes Weiterleben des Platt erhofft. Die GETAS-Befragung 1984 (vgl. dazu Goossens (1986) und Stellmacher (1987)) hat aber gezeigt, daß der Einfluß dieses Faktors nicht hoch zu veranschlagen ist: $84 \%$ der Dialektsprecher erklärten, ihr Platt im Kindesalter, und nur $9 \%$, es im Jugendalter gelernt zu haben. Seit einigen Jahren werden von Volkshochschulen und Heimatvereinen Plattdeutschkurse veranstaltet, die den Interessierten Gelegenheit geben, Dialekt zu erlernen und die Sprechfertigkeit zu üben. Dieser Unterricht der Mundart als „Fremdsprache” ist aber m.E. nicht so sehr als konservierender Faktor zu deuten, sondern vielmehr als ein Aspekt der Erscheinung „Dialekt als Kulturfaktor”. Es gibt hüben und drüben eine Dialektliteratur. Dialekt kann weiter eine bescheidene Rolle spielen in der Presse und in den gesprochenen Medien, im Unterricht, in der Kirche, in Vereinen zur Pflege der Mundart, in populärwissenschaftlichen Veröffentlichungen wie Lokalwörterbüchern. Das ist wohl im allgemeinen als eine Reaktion auf die Bedrohung der Mundart durch die Standardsprache zu deuten: Man will lokales bzw. regionales Kulturgut, mit dem man sich identifiziert, retten, was man durch Pflege zu können glaubt. Der limburgische Verein Veldeke wurde 1926 gegründet "zum Instandhalten und zur Förderung der limburgischen Mundarten"; in den jetzigen Statuten heiBt es: "Instandhalten und Förderung der Volkskultur in den beiden Provinzen Limburg, namentlich der limburgischen Mundarten". Natürlich spielen auch andere Faktoren eine Rolle, so auf deutscher Seite der sog. "niederdeutsche Sprachmythos”, also der Glaube an die alte Eigensprachlichkeit des Niederdeutschen. Die Beobachtung, daB der Dialekt auch zur Belustigung verwendet wird (in Pos- 
sen, Karnevalsschlagern, Büttenreden usw.) relativiert und differenziert das erstrebte Niveau dieses Kulturfaktors; sie widerspricht aber dieser Funktion des Dialekts nicht.

Ob diese nun wichtiger wird, je nachdem die tatsächliche Verwendung weiter zurückgeht, weib ich nicht. In Limburg wird heute zweifellos weniger Dialekt gesprochen als in den zwanziger Jahren, der Verein Veldeke mit seiner gleichnamigen Zeitschrift kennt aber eine ununterbrochene, mehr oder weniger gleichmäBige Blüte. Im Westmünsterland, wo die Mundart im Alltag noch eine erkennbare Rolle spielt, ist ihre Funktion als Kulturfaktor sicher wesentlich geringer als in der Stadt Münster, wo es - so weit ich es beurteilen kann - keine autochthonen Dialektsprecher mehr gibt. Aber gerade hier hat "das Niederdeutsche sich im Bereich der kulturellen Funktionen in den letzten Jahrzehnten einige Bereiche (...) zurück- und neuerobern können" (Peters 1992, S.63). Es leben hier münsterländische und andere westfälische Schriftsteller, es gibt an den Städtischen Bühnen eine Niederdeutsche Bühne. Es gibt die 1875 gegründete Abendgesellschaft Zoologischer Garten, die plattdeutsche Possen aufführt, mehrere plattdeutsche Amateurbühnen, Mundartsendungen in Münsterländer Platt, darunter Hörspiele, gelegentlich Fernsehsendungen in und über Plattdeutsch. In der Presse gibt es plattdeutsche Glossen und gelegentlich Anzeigen, ab und zu werden plattdeutsche Gottesdienste veranstaltet. Der Westfälische Heimatbund organisiert einen plattdeutschen Lesewettbewerb in den Schulen. Die 1983 gegründete Augustin Wibbelt-Gesellschaft hat eine Vermittlerfunktion zwischen der Wissenschaft des Niederdeutschen und der interessierten Öffentlichkeit. Das ist alles zusammengenommen nicht wenig. Doch wird man wohl nicht schließen dürfen, daß die Mundart aussterben muß, um als Kulturfaktor eine Hochblüte erleben zu können. Ihre Bedeutung in Münster hat offenbar mit einer städtischen Infrastruktur zu tun, deren breite ländliche Umgebung durch Migration heute noch genug Impulse zur Kultivierung der Mundart liefern kann. Was wird aber sein, wenn diese Umgebung durch den weiteren Rückgang der Mundart das nicht mehr leisten kann? Dann könnte auch ein Verfall ihrer kulturellen Funktionen in der Stadt einsetzen, der aber in den nächsten Jahrzehnten noch wohl nicht zu einer Lage führen wird, wie sie Haas (1995, S. 304) in seiner Besprechung des Sammelbandes von Kremer (1993) für die Stadt Genf beschrieben hat: Dort ist man nur noch in der Lage, am Nationalfeiertag „ein paar Strophen eines Liedes im seit $z$ weihundert Jahren verklungenen frankoprovenzalischen Dialekt" zu singen. 


\section{Literatur (Auswahl)}

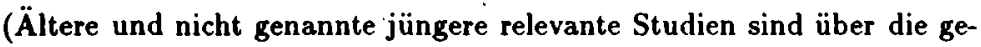
nannten Titel auffindbar)

Cornips, Leonie (1994): Syntactische variatie in het Algemeen Nederlands van Heerlen. Amsterdam.

Daan, Jo/Weijnen, Antoon (1967): "Taalsociologie”. Amsterdam. (Bijdragen en Mededelingen der Dialectencommissie van de Kon. Nederlandse Akademie van Wetenschappen 23).

Giesbers, Herman (1989): Code-switching tussen dialect en standaardtaal. Amsterdam. (Publikaties van het P.J. Meertens-Instituut 11).

Goossens, Jan (1984): Die Herausbildung der deutsch-niederländischen Sprachgrenze. Ergebnisse und Desiderate der Forschung. In: Besch, Werner/Hufeland, Klaus/Schupp, Volker/Wiehl, Peter (Hg.): Festschrift für Siegfried Grosse zum 60. Geburtstag. Gōppingen. (Gōppinger Arbeiten zur Germanistik 423). S. 23-44.

Goossens, Jan (1986): Zur Lage des Niederdeutschen und ihrer Erforschung. In: Michigan Germanic Studies 12, S. 1-20.

Goossens, Jan (1987): Het gebruik van dialect en Algemeen Nederlands en de evolutie ervan. In: Verslagen en Mededelingen van de Kon. Academie voor Nederlandse Taal en Letterkunde. S. 289-307.

Haas, Walter (1995): (Besprechung von Kremer [1993]). In: Niederdeutsches Jahrbuch 118, S. 301-304.

Hagen, Antoon (Hg.) (1986): Dialectverlies-Dialectbehoud. Taal en Tongval $38,3 / 4$, Themanummer.

Hinskens, Frans (1992): Dialect levelling in Limburg. Structural and sociolinguistic aspects. Nijmegen.

Hinskens, Frans/Hoppenbrouwers, Cor/Taeldeman, Johan ( $\mathrm{Hg}$.) (1993): Dialectverlies en regiolectvorming. Taal en Tongval, themanummer 6.

Hoppenbrouwers, Cor (1982): Language change. A study of phonemic and analogical change with particular reference to S.E. Dutch dialects. Groningen.

Hout, Roeland van (1989): De structuur van taalvariatie. Een sociolinguistisch onderzoek naar het stadsdialect van Nijmegen. Dordrecht. (Studies over taalgebruik 4).

Kremer, Ludger (1977): Mundartforschung im ostniederlāndisch-westfālischen Grenzgebiet. Eine Bestandsaufnahme (1900-1975). Amsterdam. (Beschreibende Bibliographien 7).

Kremer, Ludger (1978): Sprache und Geschichte im westfälisch-niederländischen Grenzraum. Ein Abriß der sprach-und kulturhistorischen Wechselbeziehungen. Vreden. (Beiträge des Heimatvereins Vreden zur Landes- und Volkskunde 12).

Kremer, Ludger (1979): Grenzmundarten und Mundartgrenzen. Untersuchungen zur wortgeographischen Funktion der Staatsgrenze im ostniederländisch-westfälischen Grenzgebiet. 2 Bde. Köln/Wien. (Niederdeutsche Studien 28). 
Kremer, Ludger (1983): Mundart im Westmũnsterland. Auf́bau, Gebrauch, Literatur. Borken. (Schriftenreihe des Kreises Borken 5).

Kremer, Ludger (Hg.): Diglossiestudien. Dialekt und Standardsprache im niederländisch-deutschen Grenzland. Vreden. (Westmünsterland. Quellen und Studien 1).

Kremer, Ludger/Niebaum, Hermann (Hg.) (1990): Grenzdialekte. Studien zur Entwicklung kontinentalwestgermanischer Dialektkontinua. Hildesheim/New York. (Germanistische Linguistik 101-103).

Merges, Josef Karl Christian (1977): Der untere Niederrhein. Studien zu seiner sprachlichen Entwicklung. Diss. Bonn.

Niederdeutsch und Zweisprachigkeit. Befunde, Vergleiche, Ausblicke. Beiträge zum Symposion des Instituts für niederdeutsche Sprache an der Universität Bremen 29.-31.10.1986 (1988). Leer. (Schriften des Instituts für niederdeutsche Sprache, Reihe: Dokumentation 15).

Peters, Robert (1992): Plattdeutsch in Münster und im Münsterland - gestern und heute. In: Augustin Wibbelt-Gesellschaft e.V. Jahrbuch 8, S. 43-65.

Stellmacher, Dieter (1987): Wer spricht Platt? Zur Lage des Niederdeutschen heute. Eine kurzgefaBte Bestandsaufnahme. Leer. (Schriften des Instituts fūr niederdeutsche Sprache, Reihe: Dokumentation 14).

Volkskultur an Rhein und Maas 10, 1991, 1 (Themaheft „Nachbarland Niederland ${ }^{n}$ ).

Vousten, Rob (1995): Dialect als tweede taal. Linguïstische en extra-linguistische aspecten van de verwerving van een Noordlimburgs dialect door standaardtalige jongeren. Amsterdam. 